fact, if the lens power as found by Tscherning be changed to "reduced" power it comes out even more than Gullstrand's, being $21.40 \mathrm{D}$. That is, the reduced power of the crystalline lens, using Tscherning's data for radii, indices and thickness is more by $2: 29 \mathrm{D}$. than the value found by Gullstrand.*

The method of calculating reduced surface power directly is very muctr simplified by what has been called the "dam" formula, $\mathrm{D}=\mathrm{aM}$. Here $\mathrm{D}$ stands for dioptres of power (reciprocal of "reduced" focal length) "a" stands for the difference between the two indices, (i.e. "a" amount 2 nd index is above 1st index) and $M$ stands for curvature in metrecs. The-latter is obtained by dividing the radius into 100 , or 1,000 or 40 depending upon whether the radius is given in $\mathrm{cm}$. in $\mathrm{mm}$. or in inches.

As an illustration we may apply this formula to finding the "reduced" power of the crystalline lens, using Tscherning's data which are as follows : $r 1=10.2 \mathrm{~mm}, \mathrm{r}_{2}=6.22 \mathrm{~mm} . \quad \mathrm{t}=4.06 \mathrm{~mm}$. $\mathrm{n}$ of lens $=1.42$, and $\mathrm{n}=$ of aqueous - vitreous equals 1.3365 .

For first surface $D=(1.42-\overline{1} \cdot 3365) \times \frac{1,000}{10.2}$; this works out to $8 \cdot 19$.

For second surface $D=(1.3365-1.42) \times \frac{1,000}{-6.22}$; this works out to $13 \cdot 42$.

The sum of the two surface powers is $21.61 \mathrm{D}$. The effect of thickness reduces the total power by 0.21 , giving a power of $21.40 \mathrm{D}$.

\title{
RESTORATION OF THE CHAMBER AFTER INTRA-CAPSULAR EXTRACTION
}

BY

\author{
Prof. A: Kettesy \\ DEBRECEN, HUNGARY
}

- IT is well-known, since doing intra-capsular cataract extraction that we see delayed restoration of the anterior chamber more frequently than before.

This complication in the after-treatment has generally no significance at all, more properly, less significance than in the extracapsular extraction, when there is the possibility of capsular or cortical particles left in the section. Nor has it any significance after the intra-capsular extraction either, if a shallow chamber is present if for only a short time. The well-known and easily

* The illustration taken from Duke-Elder's work is not meant as a criticism, but just to point out this omission even in the most scholarly and best known book on the subject. 
verified causes of the temporary and periodic disappearance of the anterior chamber are delayed healing, re-opening of the wound, and choroidal detachment. The eye is soft, which is a re-assuring symptom.

But it happens also-luckily very seldom-that the chamber does not form at all; this very serious sign seems not to be appreciated in detail. The anterior chamber, after a smooth intra-capsular operation, without any discoverable cause, does not reform, leading eventually to the loss of the eye." The wound is well co-apted and closed, the eye is quiet, the palpated tension is normal, Seidel's test is negative, and there is no sign of glaucoma for the first 12-14 days. Then a fine haziness of the cornea appears as the first sign of glaucoma, the tension slowly rises to $30-40 \mathrm{Hg} / \mathrm{mm}$. and without adequate medical aid the eye becomes blind.

In the last 20 years I have lost 2 eyes in this way, in spite of having performed in the second case posterior sclerotomy, cyclodialysis and trephining. The chamber could not be restored. (As I do an average of 250 cataract operations a year, including about 200 intra-capsular extractions, this makes together with the case described below 0.7 per cent. of the intra-capsulars for the last 20 years).

It was by chance that a third case showed the way to restore the anterior chamber and gave also a possible explanation. Intracapsular extraction was performed on both eyes of an old lady. It was a straightforward case, without any sign of impending trouble. The operation was uneventful in both eyes with my routine procedure : Knapp-Stanculeanu-Török, iridectomy, conjunctival hooding of the wound, akinesia and retrobulbar injection. We instil as a rule pilocarpine after intra-capsular extraction.

No restoration of the chamber occurred in either eye. There was no irritation, the wound closed, and no hypotony. We waited 6 days for spontaneous restoration.

On the seventh day we started to do something for the restoration of the chamber. We instilled 8 per cent. pilocarpine together with cocaine and adrenalin, in order to raise the tone of sphincter and dilator simultaneously,.a proceeding that leads sometimes to success: But in this case it failed, as well as repeating it with 1 per cent. adrenalin the next day.

On the ninth and tenth days we joined to the instillations intravenous injections of 40 per cent. dextrose in the hope of obtaining a result by dehydrating the vitreous, but in vain. Dionine powder into the conjunctival sac and sub-conjunctival injection of saline solution proved to be similarly inefficacious.

On the twelfth day after the operation the loss of both eyes seemed to be imminent, so we started operative interventions. Both wounds seemed to be well closed, palpated tension was normal, no lacrimation, vision good. 
In remembrance of the uselessness of posterior sclerotomy in $a^{\prime}$ similar case, I decided to do cyclodialysis on both sides, after the modification of Blaskovic's (a tergc) carried out in front of the external rectus.

The end of Heine's spatula, forcing its way during the operation into the chamber between iris and cornea, got into the pupillary area by mistake, and injured the hyaloidea. The vitreous streamed immediately forth, pushed the iris back and filled the chamber. The chamber was, restored (of course a vitreous-filled chamber). Eye-surgeons see the same thing occasionally, when, during cataract extractions the hyaloidea is broken and vitreous fills the anterior chamber.

On the fellow-eye I brought about the same event' on purpose. I pushed the point of the spatula into the pupillary area and broke the hyaloidea by a little movement sideways and backwards. The result was the same, the chamber was immediately restored.

The further course of the case was satisfactory. The palpated tension of the eye remained normal. taken by tonometer 6 days later it was $22 \mathrm{Hg} / \mathrm{mm}$. Corrected vision $6 / 9$ and 6/12. Slit-lamp examination showed the well-known signs of the vitreous-filled chamber: in the aqueous there were waving from the pupil ramifying fibres of the vitreous.

Since then I had no opportunity to perform this operation, that could be called restoration of the anterior chamber. It was only considered once, but the chamber restored itself spontaneously on the eleventh day.

The plain vitreous chamber has, as far as our experience reaches, scarcely any significance regarding post-operative glaucoma. It is true, text-books mention the loss of vitreous and the absence of the hyaloidea amongst the causes of post-operative glaucoma, but there are also plenty of observations to the contrary. Yet, admitting the possibility of bringing about the danger of later glaucoma, this is a small drawback in comparison to the direct and rapid loss of an eye after the operation.

It is interesting to note that the whole vast literature of cataract-extraction does not mention the primary, nor glaucomatous non-reappearance of the chamber, only the prolonged restoration. Of course, it must have been very rare in the pre-intracapsular period, and whenever it occurred, it was traced back to non-closure of the wound, to epithelial immigration and chiefly to an unobserved glaucomatous tendency', promoted by the operation. Though the latter possibility cannot be rejected with absolute sureness, I am convinced, in my cases there was no primary glaucomatous disposition. It seems to me more probable, the cause was purely. mechanical. The iris was flat, adhering to the cornea, the pupil 
was tightly filled with the Inelastic hyaloidea, capillary, adhesion closed up the chamber completely. Under such conditions spontaneous reversibility is hardly imaginable. The retained aqueous must be taken up by the vitreous, its swelling presses iris and hyaloidea the more to the cornea : a vicious circle thus arises which can be interrupted only by operative intervention. A similar situation is well known, of course only after glaucoma operations when it is the lens, that obturates the chamber. There is also an account of trying to restore the chamber in such cases. Asayama and Chikakiyo blow air between iris and cornea if after Elliot's trephining the chamber will not reform (Zentralblatt f. Ophthal,; Vol. XLII, p. 599).

\section{ANNOTATIONS}

\section{Neuro-paralytic Keratitis}

Although alcohol injection of the Gasserian ganglion is a strikingly successful form of treatment for trigeminal neuralgia, the patients who submit to it are always haunted by the possibility of developing lesions in the anaesthetic cornea which, if untreated, may lead to serious consequences involving, sometimes, corneal perforation, and loss of the eye. The cause of the condition has been a subject of discussion among physiblogists for many years, one group maintaining that the cornea has trophic nerve fibres which in some mysterious way look after its nutrition, and that when these fibres are blocked or severed, the cornea suffers; the other group maintains that anaesthesia of the cornea is sufficient reason for its breakdown, minute traumata occurring, unnoticed by the patient but leading to subsequent keratitis. This has always seemed to us an unsatisfactory explanation, in view of the fact that the earliest observable change in the cornea consists of the development of numerous pinpoint areas in the exposed part which stain with fluorescein, and it is difficult to imagine any form of trauma which which would produce this.

The only method of treatment until recently has been to provide - some sort of cover for the cornea. The ideal cover is, of course, the lids, and for many years now the operation of tarsorraphy has held the field, with resulting inability to use the eye. This disadvantage can be mitigated by gradually dividing the adhesions once the cornea has regained its normal state, and it is frequently found that an adhesion only $3 \mathrm{~mm}$. wide lying over the outer limbus is enough to maintain integrity of the corneal epithelium. Even this, however, constitutes a fairly conspicuous disfigurement, so other methods have been sought for. The application of a pad is dangerous 